\title{
Optimizing biologic sequencing in metastatic colorectal cancer: first line and beyond
}

\author{
Z. Jin $\mathrm{MD}^{*}$ and J.M. Hubbard $M D^{*}$
}

\begin{abstract}
Significant advances in the treatment of metastatic colorectal cancer (mcrc) since the early 2000s have led to improved clinical outcomes, including overall survival (os). When fluorouracil was the sole treatment agent for mCRC, os in phase III studies was approximately 12 months. Now, in 2019, the median os (mos) in the most recent mcrC clinical trials has been approaching 3 years. The biologic agents that target the vascular endothelial growth factor (VEGF), epithelial growth factor receptor (EGFR), human epidermal growth factor receptor 2 (HER2), PD-1, CTLA-4, NTRK, and BRAF pathways play important roles in the mCRC treatment algorithm, given their significant—sometimes dramatic-activity. Emerging data indicate that the choice of a specific biologic at a particular time (line of treatment) for specific patient populations (based on tumour characteristics) is critical. In the present review, we discuss the available evidence for optimal biologic sequencing in the management of mcrc.
\end{abstract}

Key Words Colorectal cancer, biologics, treatments

Curr Oncol. 2019 November;26(S1):S33-S42

www.current-oncology.com

\section{INTRODUCTION}

Worldwide, colorectal cancer (CRC) is the 2nd most commonly diagnosed cancer in men and the 3rd most commonly diagnosed in women. In 2018, 1.8 million new cases, with more than 860,000 deaths, were estimated ${ }^{1}$. In the United States, CRC is the 3rd most frequently diagnosed cancer overall, with 145,600 new cases of CRC expected to be diagnosed in $2019^{2}$.

More than $20 \%$ of patients with CRC present with synchronous metastatic disease at their initial diagnosis, and $50 \%-60 \%$ of patients develop metachronous metastases ${ }^{3-6}$. Approximately $56 \%$ of patients with CRC will ultimately die from their cancer ${ }^{7}$. Major advances in the treatment of metastatic CRC (mCRC) since the early 2000s significantly improved overall survival (os) in those patients. Mortality from CRC has been declining tremendously (almost 35\% from 1990 to 2007 and 53\% from 1970 to 2016) in the United States ${ }^{2,8}$. Those declines are largely attributable to earlier diagnosis because of screening tests and to improved treatment options, including new systemic chemotherapy agents (capecitabine, oxaliplatin, irinotecan, trifluridine/ tipiracil) and biologic agents targeting vascular endothelial growth factor (VEGF), epidermal growth factor receptor (EGFR), human epidermal growth factor receptor 2 (HER2),
PD-1, CTLA-4, MEK, BRAF, and NTRK. How the treatment of mCRC is optimized, especially by choice and sequencing of biologics, is critical to maximizing the benefits. In the present review, we discuss the role of biologics in the treatment of mCRC.

\section{REVIEW}

\section{First-Line Treatment}

\section{VEGF Inhibitors}

Vascular endothelial growth factor was first identified and isolated in the late 1980s and is recognized as an essential regulator of normal and abnormal blood vessel growth ${ }^{9,10}$. Later, the VEGF family expanded to include the prototype member VEGF (VEGF-A), placental growth factor, vEGF-B, VEGF-C, and VEGF-D ${ }^{11-14}$. Among those factors, VEGF-A constitutes the rate-limiting step in controlling blood vessel growth that includes tumour angiogenesis.

Bevacizumab is a humanized anti-vEGF monoclonal antibody that binds to and neutralizes all VEGF-A isoforms and its proteolytic fragments ${ }^{15,16}$. The AVF2107g trial was the first phase II clinical study to demonstrate the survival benefit of adding bevacizumab to chemotherapy ${ }^{17}$ (Table I). Compared with IFL and placebo, adding bevacizumab to 
OPTIMIZING BIOLOGIC SEQUENCING IN mCRC, Jin and Hubbard

TABLE I Key phase III studies for biologics in metastatic colorectal cancer

\begin{tabular}{|c|c|c|c|c|c|}
\hline \multirow{2}{*}{$\begin{array}{c}\text { Reference } \\
\text { (study name) }\end{array}$} & \multirow{2}{*}{$\begin{array}{l}\text { Patients } \\
\text { (n) }\end{array}$} & \multirow[t]{2}{*}{ Treatment arms } & \multicolumn{2}{|c|}{ Median survival (months) } & \multirow{2}{*}{$\begin{array}{l}\text { Hazard } \\
\text { ratio }\end{array}$} \\
\hline & & & Progression-free & Overall & \\
\hline Hurwitz et al., $2004^{17}$ & 402 & IFL plus bevacizumab & 10.6 & 20.3 & 0.66 \\
\hline (AVF2107g) & 411 & IFL & 6.2 & 15.6 & $p<0.001$ \\
\hline Saltz et al., $2008^{18}$ & 700 & FOLFOX4 or XELOX, both plus bevacizumab & 9.4 & 21.3 & 0.89 \\
\hline (NO16966) & 701 & FOLFOX4 or XELOX & 8.0 & 19.9 & $p=0.077$ \\
\hline Sobrero et al., $2008^{19}$ & 648 & Irinotecan plus cetuximab & 4.0 & 10.7 & 0.975 \\
\hline (EPIC) & 650 & Irinotecan & 2.6 & 10.0 & $p=0.71$ \\
\hline Maughan et al., $2011^{20}$ & 362 & FOLFOX or XELOX, both plus cetuximab & 8.6 & 17.0 & 1.04 \\
\hline$(\mathrm{COIN})$ & 367 & FOLFOX or XELOX & 8.6 & 17.9 & $p=0.67$ \\
\hline Van Cutsem et al., $2011^{21}$ & 316 & FOLFIRI plus cetuximab & 9.9 & 23.5 & 0.796 \\
\hline (CRYSTAL) & 350 & FOLFIRI & 8.4 & 20.0 & $p=0.0093$ \\
\hline Tveit et al., $2012^{22}$ & 97 & FLOX & 8.7 & 22.0 & 1.14 \\
\hline \multirow[t]{2}{*}{ (NORDIC VII) } & 97 & FLOX plus cetuximab & 7.9 & 20.1 & \multirow[t]{2}{*}{$p=0.48$} \\
\hline & 109 & Intermittent FLOX plus cetuximab & 7.5 & 21.4 & \\
\hline \multirow{2}{*}{$\begin{array}{l}\text { Van Cutsem et al., } 2012^{23} \\
\text { (VELOUR) }\end{array}$} & 612 & FOLFIRI plus aflibercept & 6.90 & 13.5 & \multirow{2}{*}{$\begin{array}{c}0.817 \\
p=0.0032\end{array}$} \\
\hline & 614 & FOLFIRI & 4.67 & 12.06 & \\
\hline \multirow{2}{*}{$\begin{array}{l}\text { Bennouna et al., } 2013^{24} \\
\text { (ML18147) }\end{array}$} & 409 & Chemotherapy plus bevacizumab & 5.7 & 11.2 & \multirow{2}{*}{$\begin{array}{c}0.81 \\
p=0.0062\end{array}$} \\
\hline & 411 & Chemotherapy & 4.1 & 9.8 & \\
\hline \multirow{2}{*}{$\begin{array}{l}\text { Cunningham et al., } 2013^{25} \\
\text { (AVEX) }\end{array}$} & 140 & Capecitabine plus bevacizumab & 9.1 & 20.7 & \multirow{2}{*}{$\begin{array}{c}0.79 \\
p=0.78\end{array}$} \\
\hline & 140 & Capecitabine & 5.1 & 16.8 & \\
\hline \multirow{2}{*}{$\begin{array}{l}\text { Douillard et al., 201326 } \\
\text { (PRIME) }\end{array}$} & 325 & FOLFOX4 plus panitumumab & 9.6 & 23.9 & \multirow{2}{*}{$\begin{array}{c}0.83 \\
p=0.072\end{array}$} \\
\hline & 331 & FOLFOX4 & 8.0 & 19.7 & \\
\hline \multirow{2}{*}{$\begin{array}{l}\text { Grothey et al., 201327 } \\
\text { (CORRECT) }\end{array}$} & 500 & Regorafenib & 1.9 & 6.4 & \multirow{2}{*}{$\begin{array}{c}0.77 \\
p=0.0052\end{array}$} \\
\hline & 253 & Placebo & 1.7 & 5.0 & \\
\hline \multirow{2}{*}{$\begin{array}{l}\text { Heinemann et al., } 2014^{28} \\
\text { (FIRE-3) }\end{array}$} & 297 & FOLFIRI plus cetuximab & 10.0 & 28.7 & \multirow{2}{*}{$\begin{array}{c}0.77 \\
p=0.017\end{array}$} \\
\hline & 295 & FOLFIRI plus bevacizumab & 10.3 & 25.0 & \\
\hline \multirow{2}{*}{$\begin{array}{l}\text { Loupakis et al., } 2014^{29} \\
\text { (TRIBE) }\end{array}$} & 252 & FOLFOXIRI plus bevacizumab & 12.1 & 31.0 & \multirow{2}{*}{$\begin{array}{c}0.79 \\
p=0.054\end{array}$} \\
\hline & 256 & FOLFIRI plus bevacizumab & 9.7 & 25.8 & \\
\hline \multirow{2}{*}{$\begin{array}{l}\text { Peeters et al., } 2014^{30} \\
\quad(20050181)\end{array}$} & 303 & FOLFIRI plus panitumumab & 6.7 & 14.5 & \multirow{2}{*}{$\begin{array}{c}0.92 \\
p=0.37\end{array}$} \\
\hline & 294 & FOLFIRI & 4.9 & 12.5 & \\
\hline Tabernero et al., $2015^{31}$ & 536 & FOLFIRI plus ramucirumab & 5.7 & 13.3 & 0.844 \\
\hline (RAISE) & 536 & FOLFIRI & 4.5 & 11.7 & $p=0.0219$ \\
\hline Venook et al., $2017^{32}$ & 578 & FOLFOX or FOLFIRI, both plus cetuximab & 10.5 & 30.0 & 0.88 \\
\hline (CALGB 80405) & 559 & FOLFOX or FOLFIRI, both plus bevacizumab & 10.6 & 29.0 & $p=0.08$ \\
\hline Qin et al., $2018^{33}$ & 193 & FOLFOX4 plus cetuximab & 9.2 & 20.7 & 0.76 \\
\hline (TAILOR) & 200 & FOLFOX4 & 7.4 & 17.8 & $p=0.02$ \\
\hline Cremolini et al., 2019 & 340 & FOLFOXIRI plus bevacizumab reintroduction & $19.1^{\mathrm{a}}$ & 27.6 & 0.81 \\
\hline$(\text { TRIBE2) })^{34}$ & 339 & FOLFOX plus bevacizumab, then & $16.4^{\mathrm{a}}$ & 22.6 & $p=0.033$ \\
\hline & & FOLFIRI plus bevacizumab & & & \\
\hline Kopetz et al., $2019^{35}$ & 224 & Encorafenib plus binimetinib plus cetuximab & NR & 9.0 & $0.52^{\mathrm{b}}$ \\
\hline (BEACON) & 221 & Irinotecan or FOLFIRI, both plus cetuximab & NR & 5.4 & $p<0.0001$ \\
\hline & 220 & Encorafenib plus cetuximab & NR & 8.4 & $0.60^{c}$ \\
\hline & & & & & $p=0.0003$ \\
\hline Sastre et al., $2019^{36}$ & 172 & FOLFOXIRI plus bevacizumab & 12.4 & 21.7 & $p=0.862$ \\
\hline (VISNU-1) & 177 & FOLFOX plus bevacizumab & 9.3 & 17.6 & \\
\hline
\end{tabular}

a For PFS2, defined as the time from randomization to death or to disease progression on any treatment given after 1 st disease progression.

b Encorafenib-binimetinib-cetuximab arm compared with the irinotecan-cetuximab arm.

c Encorafenib-cetuximab arm compared with the irinotecan-cetuximab arm.

IFL = irinotecan, fluorouracil, leucovorin; FOLFOX = bolus and infusional fluorouracil, leucovorin, oxaliplatin; XELOX = capecitabine, oxaliplatin; FOLFIRI = fluorouracil, leucovorin, irinotecan; FOLFOXIRI = fluorouracil, leucovorin, oxaliplatin, irinotecan; FLOX = bolus fluorouracil, leucovorin, oxaliplatin; NR = not reported. 
the IFL regimen (irinotecan-fluorouracil-leucovorin) significantly improved median os (mos), response rate (RR), and median duration of response. Subsequently, in 2004, the U.S. Food and Drug administration (FDA) approved bevacizumab to be used in combination with intravenous fluorouracil-based chemotherapy as a first-line treatment for patients with mcRC.

Meanwhile, the phase III N9741 trial revealed the clinical benefit in mos, median progression-free survival (mPFs), and RR of FOLFOX treatment (infusional fluorouracilleucovorin-oxaliplatin) compared with $\mathrm{IFL}^{37}$. Those results led to the widespread adoption of FOLFOx instead of IFL as the backbone chemotherapy regimen to be combined with bevacizumab.

However, in the NO16966 study comparing FOLFOX [or CAPOx (capecitabine-oxaliplatin)] with and without bevacizumab in the first-line treatment of unresectable mcRC, bevacizumab was associated with only a modest 1.4-month mpFs benefit (study primary endpoint) and a modest mos benefit of 1.4 months, which was not statistically significant ${ }^{18}$. One explanation for those results was the relatively short exposure of the patients to bevacizumab in the study (only $29 \%$ of patients in the bevacizumab arm received treatment until disease progression).

No randomized trials comparing FOLFIRI (fluorouracilleucovorin-irinotecan) with FOLFIRI plus bevacizumab have been reported, but a meta-analysis involving 3502 patients reported that, with FOLFIRI-bevacizumab treatment, the RR was $51.4 \%$; the mpFs, 10.8 months; and the mos, 23.7 months $^{38}$.

The open-label randomized phase III AVEX study compared the efficacy and safety of capecitabine-bevacizumab with capecitabine only in elderly patients ( $>70$ years) with untreated mcRc, enrolling 140 patients into each $\operatorname{arm}^{25}$. In the capecitabine-bevacizumab arm, mpFs was significantly longer. The study demonstrated the efficacy and safety of bevacizumab in addition to single-agent fluoropyrimidine chemotherapy in the first-line setting and showed that it is a good option for patients who are not candidates for a doublet chemotherapy backbone regimen.

The phase III TRIBE trial randomized 508 treatmentnaïve patientswith mcRcto FOLFIRI or FOLFOXIRI (fluorouracilleucovorin-oxaliplatin-irinotecan), both plus bevacizum$\mathrm{ab}^{29}$. In the FOLFOXIRI-bevacizumab arm, mPFs, RR, and mos were observed to be improved. Although the data were encouraging, the advantage of upfront triplet chemotherapy compared with a sequential strategy of doublets (FOLFOX, FOLFIRI) was uncertain, especially given the toxicities associated with intensified treatment. Thus, the phase III TRIBE2 study was conducted to confirm the benefits of a first-line triplet regimen with bevacizumab. The 679 patients with unresectable mCRC who were enrolled into TRIBE2 were randomized to either FOLFOX-bevacizumab followed by FOLFIRI-bevacizumab after disease progression (arm A) or to FOLFOXIRI-bevacizumab followed by a reintroduction of the same regimen after disease progression $(\operatorname{arm~B})^{34}$. First-line treatments were administered for up to 8 cycles, followed by fluorouracil-bevacizumab until disease progression. The primary endpoint was PFs2, defined as either death or the time from randomization to disease progression on any treatment given after the 1st disease progression. At a median follow-up of 30.6 months, arm B showed a significant advantage in PFs2 (19.1 months vs. 16.4 months) and mos (27.6 months vs. 22.6 months). Similarly, the phase III VISNU-1 trial compared FOLFOXIRIbevacizumab with Folfox-bevacizumab in the first-line setting for patients with mcrc who had 3 or more baseline circulating tumour cells ${ }^{36}$. The FOLFOXIRI-bevacizumab arm was associated with a statistically significant improvement in mPFs, the primary endpoint (12.4 months vs. 9.3 months), and a numerically higher mos (21.7 months vs. 17.6 months, $p=0.862$ ).

Several meta-analyses have confirmed the mpFs and mos benefit of bevacizumab in the first-line treatment of mCRC, although subgroup analysis has suggested that the bevacizumab-related survival benefit is observed only when bevacizumab is combined with irinotecan-based chemotherapy $^{39-42}$. It is widely accepted that the addition of bevacizumab to first-line chemotherapy offers a modest clinical benefit. The side effects associated with bevacizumab include hypertension, thromboembolic events, bleeding, proteinuria, wound complications, and gastrointestinal perforation, but this agent is well tolerated in general.

\section{EGFR Inhibitors}

Epidermal growth factor receptor plays an important role in CRC initiation and progression, and EGFR overexpression is detected in $49 \%-82 \%$ of CRCs $^{43,44}$. Cetuximab and panitumumab are monoclonal antibodies against the extracellular domain of the receptor and inhibit its downstream signalling pathways. The RAs/RAF/MAPK pathway is downstream of EGFR, and its mutations are predictive for a lack of benefit from EGFR inhibitors ${ }^{26,45,46}$. It has become clear that patients with mcrc having RAS (KRAS, NRAS) mutations will not benefit from treatment with an EGFR inhibitor ${ }^{47}$. Evidence has also strongly suggested that $B R A F$ V600E-mutated mCRC (even if $R A S$ wild-type) is unlikely to respond to an EGFR inhibitor ${ }^{48,49}$. It is crucial to perform an extended RAS mutation test (KRAS or NRAS exons 2-4) and a $B R A F$ mutation test before starting EGFR inhibitor treatment in mCRC.

The phase III CRYSTAL trial investigated the efficacy of cetuximab-FOLFIRI as a first-line treatment for MCRC $^{50}$. Patients were randomized to FOLFIRI with or without cetuximab. In the initial report, the addition of cetuximab to FOLFIRI was associated with only a modest improvement in PFS, without a benefit in os. However, in the updated analysis, the addition of cetuximab to FOLFIRI in patients with KRAS wild-type mCRC was associated with significant improvements in mos, mPFs, and $\mathbf{R R}^{21}$.

Cetuximab was also tested in combination with FOLFOX in first-line treatment for mCRC. In the randomized phase II opus trial, adding cetuximab to FoLFox was associated with an increased RR and mPFs, but without a mos benefit, in patients with KRAS wild-type disease ${ }^{51,52}$. Likewise, adding cetuximab to FOLFOX or XELOX (capecitabine-oxaliplatin) in the phase III COIN study did not demonstrate a benefit in mos or mPFs for the patients with KRAS wild-type disease $^{20}$. An improvement in mPFs was seen in the Folfox subgroup, but not in the xelox subgroup. The phase III NORDIC VII trial, in which cetuximab was added to the FLox 
(fluorouracil-oxaliplatin) regimen, also did not show a PFs or os benefit for cetuximab ${ }^{22}$.

However, the most recently reported phase III openlabel randomized TAILOR study clearly demonstrated the benefit of adding cetuximab to FoLFOx in the first-line setting ${ }^{33}$. In that study, 393 patients with mCRC (KRAS or NRAS exons 2-4 wild-type) were treated with FOLFOx with or without cetuximab. Adding cetuximab significantly improved the mPFs (primary study endpoint, 9.2 months vs. 7.4 months) and mos (20.7 months vs. 17.8 months). The efficacy of FOLFOX-cetuximab was also confirmed in the phase III Cancer and Leukemia Group B/swog 80405 trial $^{32}$. The difference between those studies raised the possibility that the chemotherapy backbone, especially the fluoropyrimidine formula, might be critical, and capecitabine-based chemotherapy was not listed for combination with cetuximab in the U.S. National Comprehensive Cancer Network guideline. Another interesting finding was that less oxaliplatinassociated peripheral neuropathy was seen in the cetuximab combination group. That observation suggested that cetuximab might have neuroprotective effects ${ }^{20,22,51,52}$.

Panitumumab was tested in combination with FOLFOX in the phase III PRIME study ${ }^{53}$. In patients with KRAS wild-type mCRC, the addition of panitumumab to FOLFOX treatment was associated with a significant improvement in mPFs (9.6 months vs. 8.0 months), but the mos improvement did not reach statistical significance (23.9 months vs. 19.7 months). Interestingly, panitumumab seemed to be detrimental when used in KRAS-mutant mCRC; however, the mechanism is still unclear. Nonetheless, that observation confirmed the importance of a comprehensive RAS mutation panel test before treatment with an EGFR inhibitor.

Cetuximab and panitumumab can both cause infusionrelated reactions (more common in cetuximab), acneiform dermatitis, diarrhea, stomatitis, and hypomagnesemia. Skin toxicities are the most common side effects with EGFR inhibitors. Pre-emptive treatment, including the use of skin moisturizers, sunscreen, topical steroids, and doxycycline, can significantly mitigate the skin toxicities ${ }^{54}$. In general, cetuximab and panitumumab are considered interchangeable in the first-line treatment for mCRC.

\section{Choosing Between VEGF and EGFR Inhibitors in the First-Line Setting}

The phase II PEAK trial compared FOLFOX-panitumumab with FOLFOX-bevacizumab in the first-line treatment of patients with KRAS exon 2 wild-type mcrC ${ }^{55}$. The mPFs and mos were found to be longer in the panitumumab arm in the subset of 170 patients who were found to have wild-type KRAS and NRAS.

The multicentre open-label randomized phase III German FIRE-3 trial compared the efficacy of FOLFIRIcetuximab with that of FOLFIRI-bevacizumab ${ }^{28}$, enrolling 593 patients with KRAS exon 2 wild-type mcrc. The study returned a negative result because the objective response rate (ORR), the primary endpoint of the study, was $62 \%$ in the cetuximab group compared with $58 \%$ in the bevacizumab group ( $p=0.18$ ). The mpFs was also similar in the cetuximab and bevacizumab groups (10.0 months vs. 10.3 months). However, the mos was significantly better in the cetuximab arm than in the bevacizumab arm (28.7 months vs. 25.0 months). In a subsequent analysis of the 400 patients with KRAS and NRAS wild-type tumours, the orR was significantly higher in the cetuximab group ( $72 \%$ vs. $56 \%)$. The mos was also significantly longer in the cetuximab group (33 months vs. 25 months $)^{56}$. Further retrospective analysis revealed that the benefit of cetuximab over bevacizumab was limited to patients with a left-sided primary tumour (mos: 38.3 months vs. 28 months); in right-sided primary tumours, bevacizumab was better (mos: 23 months vs. 18.3 months $)^{57}$.

A similar study (Cancer and Leukemia Group B/ swog 80405) conducted in the United States enrolled 1137 patients with KRAS exon 2 wild-type mCRC and treated them with FOLFOX or FOLFIRI, plus either cetuximab or bevacizumab ${ }^{32}$. The mos (study primary endpoint) and mPFs in the cetuximab arm were similar to those in the bevacizumab arm (mos: 30 months vs. 29 months; mPFs: 10.5 months vs. 10.6 months). In the all RAS wild-type patients, mos was better in cetuximab-treated leftsided primary tumours ( 37.5 months vs. 32.1 months), but bevacizumab seemed to be more beneficial in right-sided primary tumours ( 24.5 months vs. 16.4 months $)^{58}$.

A meta-analysis of the three foregoing studies revealed that, in $R A S$ wild-type left-sided colon cancer, the survival benefit was significantly greater with an EGFR inhibitor than with bevacizumab (hazard ratio: $0.71 ; p=0.0003$ ) and that patients with right-sided primary colon cancer benefited from bevacizumab, with a trend toward longer survival (hazard ratio: $1.3 ; p=0.081)^{59}$.

Based on those data, it would be reasonable to consider an EGFR inhibitor for patients with KRAS, NRAS, and $B R A F$ wild-type left-sided mCRC, while starting bevacizumab for right-sided tumours. However, patient preference, quality of life, and side effect profiles have to be considered too. Bevacizumab is generally easier to tolerate, without the skin rashes typically associated with EGFR inhibitors and with a mos similar to that in the Cancer and Leukemia Group B 80405 study. It is still reasonable to consider bevacizumab in the first-line setting for left-sided disease, using an EGFR inhibitor in later lines.

\section{Subsequent-Line Treatment}

\section{VEGF Inhibitors}

The ML18147 study and the BEBYP trial both demonstrated a mos benefit by continuing bevacizumab beyond disease progression $^{24,60}$. The randomized open-label phase III E3200 trial showed a mos benefit by adding bevacizumab to FOLFOX treatment after disease progression on an irinotecanbased first-line treatment ${ }^{61}$. Based on the data from those trials, continuing or starting bevacizumab after disease progression is known to be beneficial.

Other VEGF inhibitors include ziv-aflibercept, which is a recombinant protein designed to trap VEGF and inhibit angiogenesis. The velour study demonstrated its activity in second-line treatment for $\mathrm{mCRC}^{23}$. Ramucirumab, a human monoclonal antibody targeting VEGF receptor 2, demonstrated an os benefit in the RAISE study in the second-line setting ${ }^{31}$. However, these newer vEGF-targeted agents have undergone no head-to-head comparisons or produced dramatic survival benefits. Bevacizumab 
is still the most commonly used VEGF inhibitor in the second-line setting.

Regorafenib, which also targets the VEGF receptor, is an oral multikinase inhibitor with antiangiogenesis effects, but low specificity. The phase III international coRrect study demonstrated a 1.4-month os benefit for regorafenib compared with best supportive care in heavily pretreated patients with mCRC $^{27}$. The common side effects with regorafenib include hand-foot syndrome, fatigue, diarrhea, and hypertension. The initial approved dose of regorafenib was $160 \mathrm{mg}$ daily, 21 days on and 7 days off, but most patients were found to have difficulty tolerating treatment. The phase II regorafenib dose optimization study, ReDos, showed that, in terms of the percentage of patients who initiated a third cycle of regorafenib ( $43 \%$ vs. $24 \%$ ), weekly regorafenib dose escalation from $80 \mathrm{mg}$ to $160 \mathrm{mg}$ daily for the first cycle of treatment is superior to starting with a dose of $160 \mathrm{mg}$ daily ${ }^{62,63}$. In addition, a trend toward improved survival was observed in the dose escalation arm compared with the standard dosing arm (9.0 months vs. 5.9 months).

\section{EGFR Inhibitors}

In contrast to the situation with bevacizumab, no evidence supports continuation of an EGFR inhibitor after disease progression. However, there is strong evidence for introducing one either as a single agent or combined with chemotherapy after disease progression.

The 20050181 study confirmed a PFs benefit for panitumumab in the second-line setting, although the mos benefit was not statistically significant ${ }^{30}$. In an open-label phase III study, panitumumab also demonstrated single-agent activity in mCRC after disease progression on oxaliplatin- and irinotecan-based chemotherapy ${ }^{64}$. The retrospective subset analysis from that study after KRAS exon 2 mutation testing revealed that the panitumumab benefit was seen only in patients who were KRAS exon 2 wild-type ${ }^{65}$.

In the EPIC study, no os benefit was associated with cetuximab in the second-line setting, but mpFs and RR were significantly improved ${ }^{19}$. That finding might reflect the fact that patients were enrolled to the study without their KRAS status being known. As a single agent (compared with best supportive care), cetuximab was associated with significantly prolonged mos and PFs. Subset analysis later revealed that patients who were KRAS exon 2 wild-type benefited from cetuximab (mos: 9.5 months vs. 4.8 months; mPFs: 3.7 months vs. 1.9 months); patients with a KRAS mutation did not (hazard ratio: 0.97$)^{66}$.

Consequently, adding an EGFR inhibitor to treatment in patients with $K R A S, N R A S$, and $B R A F$ wild-type mCRC is recommended for consideration if the patient has not been exposed to the agent before.

\section{Biologics in Special Populations}

Deficient Mismatch Repair or High Microsatellite Instability mCRC: Deficiencies in the cellular mismatch repair system (dMMR) lead to DNA replication errors and high microsatellite instability (MSI-H), resulting in cancer. About $15 \%$ of patients with CRC harbour dMMR, and in about $20 \%$ of those cases, the cancers are related to Lynch syndrome, which is secondary to germline mutations ${ }^{67,68}$. Approximately $5 \%$ of patients with mCRC have dMMR disease, and $34.6 \%$ have a $B R A F$ V600E mutation, which suggests sporadic dMMR instead of Lynch syndrome ${ }^{69}$.

Immune checkpoints are key regulators of the immune system. Checkpoint proteins expressed by various cells, including tumour cells, block active immune surveillance. Checkpoint inhibitors restore immune system function by targeting those proteins.

In a phase II study, 28 patients with mcRc were treated with pembrolizumab, a humanized PD-1 monoclonal antibody ${ }^{70}$. In the 10 patients with dMMR, the response rate was $40 \%$ and the disease control rate was $90 \%$. In contrast, no response was observed in patients with proficient MMR mcrc. The U.S. FDA approved pembrolizumab after a fluoropyrimidine, oxaliplatin, and irinotecan for patients with dMMR mCRC.

The open-label phase II CheckMate 142 study had 6 experimental $\mathrm{arms}^{71}$. In the nivolumab (a PD-1 monoclonal antibody) monotherapy arm, 74 patients with dMMR mCRC who had received at least 1 line of treatment were enrolled. The investigator-assessed oRR was $31.1 \%$ (23 of 74 patients), and $69 \%$ of the patients $(n=51)$ experienced disease control for 12 or more weeks ${ }^{71}$. In the nivolumab-ipilimumab (a CTLA- 4 antibody) combination arm, 119 patients with dMMR mCRC were treated for 4 cycles and then received nivolumab as a single agent every 2 weeks until disease progression ${ }^{72}$. The orR was $55 \%$, with $80 \%$ of patients achieving disease control for 12 or more weeks. The PFs was $76 \%$ at 9 months and $71 \%$ at 12 months. Nivolumab was also approved by the U.S. FDA for the treatment of dMMr mCrC after chemotherapy with or without ipilimumab.

BRAF V600E Mutated mCRC: In the EGFR/RAs/RAF/ MEK/ERK pathway, BRAF is a key player. The $B R A F$ V600E mutation $\left(B R A F^{\mathrm{V} 600 \mathrm{E}}\right)$ causes constitutive activation of MAPK signalling. Approximately $8 \%$ of patients with CRC harbour $B R A F^{\mathrm{V} 600 \mathrm{E}}$, which has distinct clinicopathologic characteristics $^{73,74}$. Cases of $B R A F^{\mathrm{V} 600 \mathrm{E}}$ mcRC are more commonly seen in older female patients and typically have poor clinical outcomes $20,75,76$.

Approximately $20 \%$ of patients with $B R A F^{\mathrm{V} 600 \mathrm{E}} \mathrm{mCRC}$ also have dMMR or MSI-H, and immune checkpoint inhibitor treatments have proved to be effective for those patients ${ }^{71,72}$. However, most patients with $B R A F^{\mathrm{V} 600 \mathrm{E}}$ mCRC have proficient MMR, and their disease progresses rapidly with standard chemotherapy. Intensified chemotherapy with FOLFOXIRI-bevacizumab seems to be beneficial in this subset of patients based on an analysis of the TRIBE study $^{77}$. Of 28 patients with $B R A F^{\mathrm{V} 600 \mathrm{E}}$ mCRC in the TRIBE study, median os was better in the FOLFOXIRI-bevacizumab arm (16 patients) than in the FOLFIRI-bevacizumab arm (19.0 months vs. 10.7 months).

It was learned from early studies that a BRAF inhibitor does not have significant single-agent activity in $B R A F^{\mathrm{V} 600 \mathrm{E}}$ mCRC, which might be attributable to the feedback activation of EGFR signalling ${ }^{78-80}$. Combining an EGFR inhibitor with a BRAF inhibitor was subsequently tested in the swoG 1406 study, which enrolled 106 patients with $B R A F^{\mathrm{V} 600 \mathrm{E}}$ extended-KRAS wild-type mCRC. Patients were treated with irinotecan-cetuximab with or without vemurafenib (a BRAF inhibitor) ${ }^{81}$. A better mPFs (primary endpoint, 4.4 months vs. 2.0 months), ORR ( $16 \%$ vs. $4 \%$ ), and disease control rate 
( $67 \%$ vs. $22 \%$ ) were observed in the vemurafenib arm compared with the no-vemurafenib arm.

Given the critical role of downstream MAPK signalling in $B R A F^{\mathrm{V} 600 \mathrm{E}} \mathrm{mCRC}$ and the successes observed from the combination of a BRAF inhibitor with a MEK inhibitor in melanoma treatment ${ }^{82,83}$, a similar approach was also tested in $B R A F^{\mathrm{V} 600 \mathrm{E}}$ mCRC. In a phase I/II clinical trial, triplet treatment with dabrafenib (a BRAF inhibitor), trametinib (a MEK inhibitor), and panitumumab was associated with a higher response rate than was seen with panitumumabdabrafenib (21\% vs. $10 \%)$. No response was detected in the panitumumab-trametinib arm ${ }^{84}$.

The randomized open-label phase III BEACON trial was designed to further test the efficacy of a novel triplet treatment [binimetinib (a MEK inhibitor), encorafenib (a BRAF inhibitor), and cetuximab] for previously treated $B R A F^{\mathrm{V} 600 \mathrm{E}}$ mCRC $^{85}$. The study had a safety lead-in phase in which 30 patients were enrolled and treated with that triplet. The data from those 30 patients confirmed an oRR of $48 \%$, with a mPFs of 8.0 months and a mos of 15.3 months, all of which were significantly better than results in historical controls. The preliminary result from the phase III study again confirmed an improved mos (9.0 months vs. 5.4 months) and ORR ( $26 \%$ vs. $2 \%$ ) when the triplet was compared with irinotecan-cetuximab treatment ${ }^{35}$. The triplet treatment is now considered to be the new standard treatment for $B R A F^{\mathrm{V} 600 \mathrm{E}}$ mCRC.

HER2-Positive mCRC: The HER2 member of the human EGFR family has been a successful treatment target in breast cancer, esophageal adenocarcinoma, and gastroesophageal junction adenocarcinoma. Overexpression of HER2 in CRC is rare, but its prevalence is higher (5\%-14\%) in KRAS, $N R A S$, and $B R A F$ wild-type $\operatorname{mCRC}^{86,87}$.

The Italian open-label phase II HERACLES study enrolled 27 patients with KRAS exon 2 wild-type HER2-positive mCRC $^{86}$. The patients were heavily treated before study enrolment, having received an average of 4 previous lines of treatment. Treatment with trastuzumab (a HER2 antibody) and lapatinib (a HER2 tyrosine kinase inhibitor) was associated with an oRR of $30 \%$ (8 patients), with 1 patient experiencing a complete response and 12 patients $(44 \%)$ having stable disease. Recently, the MyPathway study reported on dual HER2 antibody inhibition with pertuzumab and trastuzumab in 57 patients with HER2-overexpressing mCRC $^{88}$. The ORR was $32 \%$ (18 patients), with 1 patient experiencing a complete response. Most of the patients were KRAS wild-type (75\%) and had received multiple previous lines of treatment.

Given the encouraging results from the foregoing studies, the U.S. National Comprehensive Cancer Network guideline lists HER2-targeted treatment as an option for HER2-overexpressing mCRC.

NTRK Fusion mCRC: The NTRK family consists of 3 members that are encoded by the NTRK1, NTRK2, and NTRK3 genes. Gene fusions involving those genes lead to constitutively activated NTRK protein and tumorigenesis ${ }^{89}$. Colorectal cancer is one of the first cancer types in which a NTRK gene translocation (TPM3-NTRK1) was detected (in 1986) ${ }^{90}$, but $N T R K$-fusion CRC did not capture a lot of attention until recently, when novel therapies targeting the associated pathway showed remarkable antitumour activity $^{91}$. The small subset of CRCs that harbour a NTRK fusion gene $(0.1 \%-1 \%)$ might benefit from larotrectinib, a highly selective small-molecule pan- NTRK inhibitor ${ }^{92,93}$. An early-phase study of larotrectinib treatment enrolled 55 patients with 17 unique $N T R K$ fusion-positive cancers (including 4 colon cancers) ${ }^{94}$. In that population, the NTRK fusions involved all 3 family members, and 14 unique upstream fusion partners were detected. Larotrectinib was associated with an ORR of $75 \%$ by independent radiology review. Most of the responses were durable (median duration of response was not reached at a median follow-up of 8.3 months), and $71 \%$ of responses were ongoing at 1 year. Given the significant clinical benefits, larotrectinib is approved by the U.S. FDA for the treatment of NTRK fusion-positive solid tumours and has been incorporated into the CRC treatment guideline from the U.S. National Comprehensive Cancer Network.

\section{SUMMARY}

Worldwide, mcrc is a lethal disease, although the mortality rate is improving with recent advancements in treatment. In the first and subsequent lines of treatment, biologics play a very important role. The choice of biologics and their sequencing depend largely on patient factors (preferences, age, performance status, etc.) and disease characteristics [disease sidedness; KRAS, NRAS, and BRAF mutation status; MSI status; HER2 overexpression, etc. (Figure 1)]. To
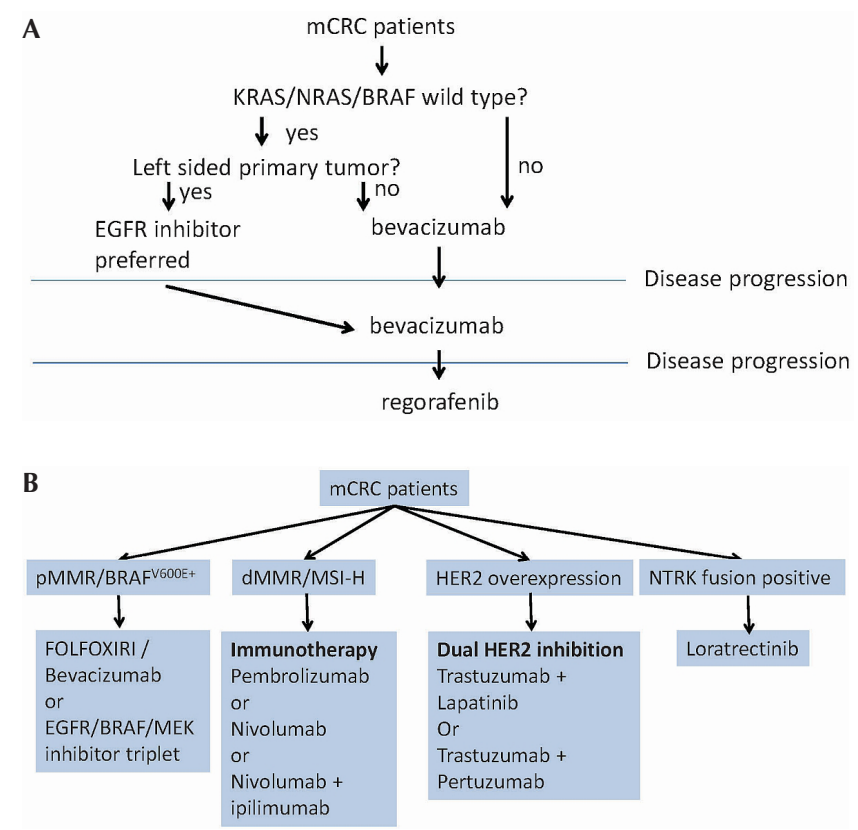

FIGURE 1 (A) Simplified consideration for choosing biologics. (B) Biologics for specific patient populations.

$\mathrm{mCRC}=$ metastatic colorectal cancer; $\mathrm{EGFR}=$ epidermal growth factor receptor; $\mathrm{pMMR}=$ proficient mismatch repair; $\mathrm{dMMR}=$ deficient mismatch repair; MSI-H = high microsatellite instability; HER2 = human epidermal growth factor receptor 2 ; FOLFOXIRI = fluorouracil-leucovorinoxaliplatin-irinotecan. 
maximize survival benefits, it is crucial that patients with mCRC are exposed to all potentially active medications during the course of their treatment.

Although most patients with mCRC will start with a doublet chemotherapy backbone plus a biologic, young and symptomatic patients could benefit from upfront FOLFOXIRI-bevacizumab. Fluoropyrimidine-bevacizumab might be more appropriate for frail patients. Data support the use of first-line EGFR inhibitors for left-sided mCRC that is $K R A S, N R A S$, and $B R A F$ wild-type; bevacizumab can be used for both left- and right-sided mcRc. Continuation of bevacizumab, but not EGFR inhibitors, has demonstrated a modest survival benefit after first-line disease progression. The EGFR inhibitors have single-agent activity; bevacizumab lacks data to support its use as a single agent for later lines of treatment. Immune checkpoint inhibitors are critical for the treatment of patients with dMMR (MSI-H) MCRC in both Lynch syndrome and sporadic mutation cases. The novel triplet combination of an EGFR inhibitor, a MEK inhibitor, and a BRAF inhibitor should be incorporated into daily practice for $B R A F^{\mathrm{V} 600 \mathrm{E}}$ mCRC. Dual HER2 inhibition with trastuzumab-lapatinib or trastuzumab-pertuzumab for HER2-overexpressing MCRC has some very promising preliminary data, and ongoing studies will likely lead to regulatory approval of that strategy.

The advancement of cancer treatments in CRC is based on a better understanding of tumour biology and the tumour microenvironment. Molecular analysis of each patient's tumour by next-generation sequencing upon diagnosis is recommended to determine the optimal systemic therapy combinations throughout the course of the patient's disease. That approach will also identify additional mutations or alterations and might identify clinical trial opportunities involving the next generation of biologic therapies.

\section{CONFLICT OF INTEREST DISCLOSURES}

We have read and understood Current Oncology's policy on disclosing conflicts of interest, and we declare the following interests: JMH has served as an advisory board member for Bayer Pharmaceuticals and her institution receives funding from Merck, Taiho Oncology, and Bayer Pharmaceuticals for trials in which she is an investigator. ZJ has no conflicts of interest to disclose.

\section{AUTHOR AFFILIATIONS}

*Department of Medical Oncology, Mayo Clinic, 200 First Street SW, Rochester, Minnesota 55905 U.S.A.

\section{REFERENCES}

1. World Health Organization, Union for International Cancer Control (uicc). New Global Cancer Data: GLOBocan 2018 [Web resource]. Geneva, Switzerland: Uicc; 2019. [Available at: https://www.uicc.org/news/new-global-cancer-dataglobocan-2018; cited 14 October 2019]

2. Siegel RL, Miller KD, Jemal A. Cancer statistics, 2019. $C A$ Cancer J Clin 2019;69:7-34.

3. van der Geest LG, Lam-Boer J, Koopman M, Verhoef C, Elferink MA, de Wilt JH. Nationwide trends in incidence, treatment and survival of colorectal cancer patients with synchronous metastases. Clin Exp Metastasis 2015;32:457-65.

4. Lee WS, Yun SH, Chun HK, et al. Pulmonary resection for metastases from colorectal cancer: prognostic factors and survival. Int J Colorectal Dis 2007;22:699-704.
5. Van Cutsem E, Nordlinger B, Adam R, et al. on behalf of the European Colorectal Metastases Treatment Group. Towards a pan-European consensus on the treatment of patients with colorectal liver metastases. Eur J Cancer 2006;42:2212-21.

6. Yoo PS, Lopez-Soler RI, Longo WE, Cha CH. Liver resection for metastatic colorectal cancer in the age of neoadjuvant chemotherapy and bevacizumab. Clin Colorectal Cancer 2006;6:202-7.

7. Riihimaki M, Thomsen H, Sundquist K, Hemminki K. Colorectal cancer patients: what do they die of? Frontline Gastroenterol 2012;3:143-9.

8. Siegel R, Ward E, Brawley O, Jemal A. Cancer statistics, 2011: the impact of eliminating socioeconomic and racial disparities on premature cancer deaths. CA Cancer J Clin 2011;61:212-36.

9. Leung DW, Cachianes G, Kuang WJ, Goeddel DV, Ferrara N. Vascular endothelial growth factor is a secreted angiogenic mitogen. Science 1989;246:1306-9.

10. Keck PJ, Hauser SD, Krivi G, et al. Vascular permeability factor, an endothelial cell mitogen related to PDGF. Science 1989;246:1309-12.

11. Maglione D, Guerriero V, Viglietto G, Delli-Bovi P, Persico MG. Isolation of a human placenta cDNA coding for a protein related to the vascular permeability factor. Proc Natl Acad Sci U S A 1991;88:9267-71.

12. Olofsson B, Pajusola K, Kaipainen A, et al. Vascular endothelial growth factor B, a novel growth factor for endothelial cells. Proc Natl Acad Sci U S A 1996;93:2576-81.

13. Joukov V, Pajusola K, Kaipainen A, et al. A novel vascular endothelial growth factor, VEGF-c, is a ligand for the Flt4 (VEGFR-3) and KDR (VEGFR-2) receptor tyrosine kinases. EMBO $J$ 1996;15:1751.

14. Orlandini M, Marconcini L, Ferruzzi R, Oliviero S. Identification of a c-Fos-induced gene that is related to the plateletderived growth factor/vascular endothelial growth factor family. Proc Natl Acad Sci U S A 1996;93:11675-80.

15. Muller YA, Chen Y, Christinger HW, et al. vegF and the Fab fragment of a humanized neutralizing antibody: crystal structure of the complex at 2.4 A resolution and mutational analysis of the interface. Structure 1998;6:1153-67.

16. Ferrara N, Hillan KJ, Gerber HP, Novotny W. Discovery and development of bevacizumab, an anti-vEGF antibody for treating cancer. Nat Rev Drug Discov 2004;3:391-400.

17. Hurwitz H, Fehrenbacher L, Novotny W, et al. Bevacizumab plus irinotecan, fluorouracil, and leucovorin for metastatic colorectal cancer. N Engl J Med 2004;350:2335-42.

18. Saltz LB, Clarke S, Diaz-Rubio E, et al. Bevacizumab in combination with oxaliplatin-based chemotherapy as firstline therapy in metastatic colorectal cancer: a randomized phase III study. J Clin Oncol 2008;26:2013-19.

19. Sobrero AF, Maurel J, Fehrenbacher L, et al. EPIC: phase III trial of cetuximab plus irinotecan after fluoropyrimidine and oxaliplatin failure in patients with metastatic colorectal cancer. J Clin Oncol 2008;26:2311-19.

20. Maughan TS, Adams RA, Smith CG, et al. Addition of cetuximab to oxaliplatin-based first-line combination chemotherapy for treatment of advanced colorectal cancer: results of the randomised phase 3 MRC CoIN trial. Lancet 2011;377:2103-14.

21. Van Cutsem E, Kohne CH, Lang I, et al. Cetuximab plus irinotecan, fluorouracil, and leucovorin as first-line treatment for metastatic colorectal cancer: updated analysis of overall survival according to tumor KRAS and BRAF mutation status. J Clin Oncol 2011;29:2011-19.

22. Tveit KM, Guren T, Glimelius B, et al. Phase III trial of cetuximab with continuous or intermittent fluorouracil, leucovorin, and oxaliplatin (Nordic FLOx) versus fLOx alone in first-line treatment of metastatic colorectal cancer: the NORDIC-VII study. J Clin Oncol 2012;30:1755-62. 
23. Van Cutsem E, Tabernero J, Lakomy R, et al. Addition of aflibercept to fluorouracil, leucovorin, and irinotecan improves survival in a phase III randomized trial in patients with metastatic colorectal cancer previously treated with an oxaliplatin-based regimen. J Clin Oncol 2012;30:3499-506.

24. Bennouna J, Sastre J, Arnold D, et al. on behalf of the ML18147 study investigators. Continuation of bevacizumab after first progression in metastatic colorectal cancer (ML18147): a randomised phase 3 trial. Lancet Oncol 2013;14:29-37.

25. Cunningham D, Lang I, Marcuello E, et al. on behalf of the AVEX study investigators. Bevacizumab plus capecitabine versus capecitabine alone in elderly patients with previously untreated metastatic colorectal cancer (AVEX): an open-label, randomised phase 3 trial. Lancet Oncol 2013;14:1077-85.

26. Douillard JY, Oliner KS, Siena S, et al. Panitumumab-FoLfox4 treatment and RAS mutations in colorectal cancer. $N$ Engl J Med 2013;369:1023-34.

27. Grothey A, Van Cutsem E, Sobrero A, et al. on behalf of the CORRECT study group. Regorafenib monotherapy for previously treated metastatic colorectal cancer (CORRECT): an international, multicentre, randomised, placebo-controlled, phase 3 trial. Lancet 2013;381:303-12.

28. Heinemann V, von Weikersthal LF, Decker T, et al. FOLFIRI plus cetuximab versus FOLFIRI plus bevacizumab as firstline treatment for patients with metastatic colorectal cancer (FIRE-3): a randomised, open-label, phase 3 trial. Lancet Oncol 2014;15:1065-75.

29. Loupakis F, Cremolini C, Masi G, et al. Initial therapy with FOLFOXIRI and bevacizumab for metastatic colorectal cancer. N Engl J Med 2014;371:1609-18.

30. Peeters M, Price TJ, Cervantes A, et al. Final results from a randomized phase 3 study of FOLFIRI $\{+/-\}$ panitumumab for second-line treatment of metastatic colorectal cancer. Ann Oncol 2014;25:107-16.

31. Tabernero J, Yoshino T, Cohn AL, et al. on behalf of the RAISE study investigators. Ramucirumab versus placebo in combination with second-line FOLFIRI in patients with metastatic colorectal carcinoma that progressed during or after first-line therapy with bevacizumab, oxaliplatin, and a fluoropyrimidine (RAISE): a randomised, double-blind, multicentre, phase 3 study. Lancet Oncol 2015;16:e262.

32. Venook AP, Niedzwiecki D, Lenz HJ, et al. Effect of first-line chemotherapy combined with cetuximab or bevacizumab on overall survival in patients with KRAS wild-type advanced or metastatic colorectal cancer: a randomized clinical trial. JAMA 2017;317:2392-401.

33. Qin S, Li J, Wang L, et al. Efficacy and tolerability of first-line cetuximab plus leucovorin, fluorouracil, and oxaliplatin (FOLFOX-4) versus FOLFOX-4 in patients with RAS wild-type metastatic colorectal cancer: the open-label, randomized, phase III TAILOR trial. J Clin Oncol 2018;36:3031-9.

34. Cremolini C, Antoniotti C, Lonardi S, et al. Updated results of TRIBE2, a phase III, randomized strategy study by GONO in the first- and second-line treatment of unresectable mcrc [abstract 3508]. J Clin Oncol 2019;37:. [Available online at: https://ascopubs.org/doi/abs/10.1200/JCO.2019.37.15_ suppl.3508; cited 30 August 2019]

35. Kopetz S, Grothey A, Van Cutsem E, et al. BEACON CRC: a randomized, 3-arm, phase 3 study of encorafenib and cetuximab with or without binimetinib vs. choice of either irinotecan or FOLFIRI plus cetuximab in BRAF V600E-mutant metastatic colorectal cancer [abstract LBA-006]. Ann Oncol 2019;30 (suppl 4):. [Available online at: https://academic.oup.com/ annonc/article/30/Supplement_4/mdz183.004/5526665; cited 30 August 2019]

36. Sastre J, Vieitez JM, Gomez-España MA, et al. Randomized phase III study comparing FOLFOX + bevacizumab versus
FOLFOXIRI + bevacizumab (BEv) as 1st line treatment in patients with metastatic colorectal cancer (mcrc) with $\geq 3$ baseline circulating tumor cells (bcTcs) [abstract 3507].JClin Oncol 2019;37:. [Available online at: https://ascopubs.org/ doi/abs/10.1200/JCO.2019.37.15_suppl.3507; cited 30 August 2019]

37. Goldberg RM, Sargent DJ, Morton RF, etal. A randomized controlled trial of fluorouracil plus leucovorin, irinotecan, and oxaliplatin combinations in patients with previously untreated metastatic colorectal cancer. J Clin Oncol 2004;22:23-30.

38. PetrelliF, Borgonovo K, Cabiddu M, etal. FOLFIRI-bevacizumab as first-line chemotherapy in 3500 patients with advanced colorectal cancer: a pooled analysis of 29 published trials. Clin Colorectal Cancer 2013;12:145-51.

39. Macedo LT, da Costa Lima AB, Sasse AD. Addition of bevacizumab to first-line chemotherapy in advanced colorectal cancer: a systematic review and meta-analysis, with emphasis on chemotherapy subgroups. BMC Cancer 2012;12:89.

40. Meyerhardt JA, Li L, Sanoff HK, Carpenter W 4th, Schrag D. Effectiveness of bevacizumab with first-line combination chemotherapy for Medicare patients with stage iv colorectal cancer. J Clin Oncol 2012;30:608-15.

41. Hartmann H, Muller J, Marschner N. Is there a difference in demography and clinical characteristics in patients treated with and without bevacizumab? JClin Oncol2012;30:3317-18.

42. Hurwitz HI, Lyman GH. Registries and randomized trials in assessing the effects of bevacizumab in colorectal cancer: is there a common theme? J Clin Oncol 2012;30:580-1.

43. McKay JA, Murray LJ, Curran S, et al. Evaluation of the epidermal growth factor receptor (EGFR) in colorectal tumours and lymph node metastases. Eur J Cancer 2002;38:2258-64.

44. Spano JP, Lagorce C, Atlan D, et al. Impact of EGFR expression on colorectal cancer patient prognosis and survival. Ann Oncol 2005;16:102-8.

45. Sorich MJ, Wiese MD, Rowland A, Kichenadasse G, McKinnon RA, Karapetis CS. Extended RAS mutations and anti-EGFR monoclonal antibody survival benefit in metastatic colorectal cancer: a meta-analysis of randomized, controlled trials. Ann Oncol 2015;26:13-21.

46. Douillard JY, Rong A, Sidhu R. RAS mutations in colorectal cancer. N Engl J Med 2013;369:2159-60.

47. Peeters M, Oliner KS, Price TJ, et al. Analysis of KRAS/NRAS mutations in a phase III study of panitumumab with FOLFIRI compared with FOLFIRI alone as second-line treatment for metastatic colorectal cancer. Clin Cancer Res 2015;21:5469-79.

48. Rowland A, Dias MM, Wiese MD, et al. Meta-analysis of BRAF mutation as a predictive biomarker of benefit from anti-EGFR monoclonal antibody therapy for $R A S$ wild-type metastatic colorectal cancer. BrJ Cancer 2015;112:1888-94.

49. Pietrantonio F, Petrelli F, Coinu A, et al. Predictive role of $B R A F$ mutations in patients with advanced colorectal cancer receiving cetuximab and panitumumab: a meta-analysis. Eur J Cancer 2015;51:587-94.

50. Van Cutsem E, Kohne CH, Hitre E, et al. Cetuximab and chemotherapy as initial treatment for metastatic colorectal cancer. N Engl J Med 2009;360:1408-17.

51. Bokemeyer C, Bondarenko I, Hartmann JT, et al. Efficacy according to biomarker status of cetuximab plus FOLFOX- 4 as first-line treatment for metastatic colorectal cancer: the opus study. Ann Oncol 2011;22:1535-46.

52. Bokemeyer C, Bondarenko I, Makhson A, et al. Fluorouracil, leucovorin, and oxaliplatin with and without cetuximab in the first-line treatment of metastatic colorectal cancer. JClin Oncol 2009;27:663-71.

53. Douillard JY, Siena S, Cassidy J, et al. Randomized, phase III trial of panitumumab with infusional fluorouracil, leucovorin, and oxaliplatin (FOLFOX4) versus FOLFOX4 alone as 
first-line treatment in patients with previously untreated metastatic colorectal cancer: the PRIME study. J Clin Oncol 2010;28:4697-705.

54. Lacouture ME, Mitchell EP, Piperdi B, et al. Skin Toxicity Evaluation Protocol with Panitumumab (STEPP), a phase II, open-label, randomized trial evaluating the impact of a pre-emptive skin treatment regimen on skin toxicities and quality of life in patients with metastatic colorectal cancer. JClin Oncol 2010;28:1351-7.

55. Rivera F, Karthaus M, Hecht JR, et al. Final analysis of the randomised PEAK trial: overall survival and tumour responses during first-line treatment with mFolfox6 plus either panitumumab or bevacizumab in patients with metastatic colorectal carcinoma. Int J Colorectal Dis 2017;32:1179-90.

56. Stintzing S, Modest DP, Rossius L, et al. on behalf of the FIRE-3 investigators. FOLFIRI plus cetuximab versus FOLFIRI plus bevacizumab for metastatic colorectal cancer (FIRE-3): a post-hoc analysis of tumour dynamics in the final $R A S$ wild-type subgroup of this randomised open-label phase 3 trial. Lancet Oncol 2016;17:1426-34.

57. Tejpar S, Stintzing S, Ciardiello F, et al. Prognostic and predictive relevance of primary tumor location in patients with $R A S$ wild-type metastatic colorectal cancer: retrospective analyses of the CRYSTAL and FIRE-3 trials. JAMA Oncol 2017;3:194-201.

58. Venook AP, Niedzwiecki D, Innocenti F, et al. Impact of primary $\left(1^{\circ}\right)$ tumor location on overall survival (os) and progression-free survival (PFS) in patients (pts) with metastatic colorectal cancer (mCRC): analysis of CALGB/swOG 80405 (Alliance) [abstract 3504]. J Clin Oncol 2016;34:. [Available online at: https://ascopubs.org/doi/10.1200/ JCO.2016.34.15_suppl.3504; cited 30 August 2019]

59. Holch JW, Ricard I, Stintzing S, Modest DP, Heinemann V. The relevance of primary tumour location in patients with metastatic colorectal cancer: a meta-analysis of first-line clinical trials. Eur J Cancer 2017;70:87-98.

60. Masi G, Salvatore L, Boni L, et al. Continuation or reintroduction of bevacizumab beyond progression to first-line therapy in metastatic colorectal cancer: final results of the randomized BEBYP trial. Ann Oncol 2015;26:724-30.

61. Giantonio BJ, Catalano PJ, Meropol NJ, et al. on behalf of the Eastern Cooperative Oncology Group Study E3200. Bevacizumab in combination with oxaliplatin, fluorouracil, and leucovorin (FOLFOX4) for previously treated metastatic colorectal cancer: results from the Eastern Cooperative Oncology Group Study E3200. J Clin Oncol 2007;25:1539-44.

62. Bekaii-Saab TS, Ou FS, Anderson DM, et al. Regorafenib dose optimization study (ReDOs): randomized phase II trial to evaluate dosing strategies for regorafenib in refractory metastatic colorectal cancer (mCRC). An ACCRU Network study [abstract 611]. J Clin Oncol 2018;36:. [Available online at: https:// ascopubs.org/doi/abs/10.1200/JCO.2018.36.4_suppl.611; cited 30 August 2019]

63. Bekaii-Saab TS, Ou FS, Ahn DH, et al. Regorafenib doseoptimisation in patients with refractory metastatic colorectal cancer (ReDos): a randomised, multicentre, open-label, phase 2 study. Lancet Oncol 2019;20:1070-82.

64. Van Cutsem E, Peeters M, SienaS, et al. Open-label phase III trial of panitumumab plus best supportive care compared with best supportive care alone in patients with chemotherapyrefractory metastatic colorectal cancer. J Clin Oncol 2007;25:1658-64.

65. Amado RG, Wolf M, Peeters M, et al. Wild-type KRAS is required for panitumumab efficacy in patients with metastatic colorectal cancer. J Clin Oncol 2008;26:1626-34.

66. Karapetis CS, Khambata-Ford S, Jonker DJ, et al. K-ras mutations and benefit from cetuximab in advanced colorectal cancer. N Engl J Med 2008;359:1757-65.
67. Lynch HT, de la Chapelle A. Hereditary colorectal cancer. $N$ Engl J Med 2003;348:919-32.

68. Aaltonen LA, Salovaara R, Kristo P, et al. Incidence of hereditary nonpolyposis colorectal cancer and the feasibility of molecular screening for the disease. $N$ Engl J Med 1998;338:1481-7.

69. Venderbosch S, Nagtegaal ID, Maughan TS, et al. Mismatch repair status and $B R A F$ mutation status in metastatic colorectal cancer patients: a pooled analysis of the cairo, cairo2, coin, and focus studies. Clin Cancer Res 2014;20:5322-30.

70. Le DT, Uram JN, Wang H, et al. PD-1 blockade in tumors with mismatch-repair deficiency. NEnglJ Med 2015;372:2509-20.

71. Overman MJ, McDermott R, Leach JL, et al. Nivolumab in patients with metastatic DNA mismatch repair-deficient or microsatellite instability-high colorectal cancer (CheckMate 142): an open-label, multicentre, phase 2 study. Lancet Oncol 2017;18:1182-91.

72. Overman MJ, Lonardi S, Wong KYM, et al. Durable clinical benefit with nivolumab plus ipilimumab in DNA mismatch repair-deficient/microsatellite instability-high metastatic colorectal cancer. J Clin Oncol 2018;36:773-9.

73. Davies H, Bignell GR, Cox C, et al. Mutations of the BRAF gene in human cancer. Nature 2002;417:949-54.

74. Loupakis F, Ruzzo A, Cremolini C, et al. KRAS codon 61, 146 and $B R A F$ mutations predict resistance to cetuximab plus irinotecan in KRAS codon 12 and 13 wild-type metastatic colorectal cancer. Br J Cancer 2009;101:715-21.

75. Stintzing S, Miller-Phillips L, Modest DP, et al. Impact of $B R A F$ and RAS mutations on first-line efficacy of FOLFIRI plus cetuximab versus FOLFIRI plus bevacizumab: analysis of the FIRE-3 (AIO KRK-0306) study. Eur J Cancer 2017;79:50-60.

76. Tran B, Kopetz S, Tie J, et al. Impact of BRAF mutation and microsatellite instability on the pattern of metastatic spread and prognosis in metastatic colorectal cancer. Cancer 2011;117:4623-32.

77. Cremolini C, Loupakis F, Antoniotti C, et al. FolfoxiRI plus bevacizumab versus FolfiRI plus bevacizumab as first-line treatment of patients with metastatic colorectal cancer: updated overall survival and molecular subgroup analyses of the open-label, phase 3 TRIBE study. Lancet Oncol 2015;16:1306-15.

78. Kopetz S, Desai J, Chan E, et al. Phase in pilot study of vemurafenib in patients with metastatic $B R A F$-mutated colorectal cancer. J Clin Oncol 2015;33:4032-8.

79. Prahallad A, Sun C, Huang S, et al. Unresponsiveness of colon cancer to $B R A F^{\mathrm{V} 600 \mathrm{E}}$ inhibition through feedback activation of EGFR. Nature 2012;483:100-3.

80. Corcoran RB, Ebi H, Turke AB, et al. EgFR-mediated reactivation of MAPK signaling contributes to insensitivity of $B R A F$ mutant colorectal cancers to RAF inhibition with vemurafenib. Cancer Discov 2012;2:227-35.

81. Kopetz S, McDonough SL, Morris VK, et al. Randomized trial of irinotecan and cetuximab with or without vemurafenib in $B R A F$-mutant metastatic colorectal cancer (swog 1406) [abstract 520]. J Clin Oncol 2017;35:. [Available online at: https://ascopubs.org/doi/10.1200/JCO.2017.35.4_suppl.520; cited 30 August 2019]

82. Dummer R, Ascierto PA, Gogas HJ, et al. Encorafenib plus binimetinib versus vemurafenib or encorafenib in patients with $B R A F$-mutant melanoma (columbus): a multicentre, open-label, randomised phase 3 trial. Lancet Oncol 2018;19:603-15.

83. Planchard D, Besse B, Groen HJM, et al. Dabrafenib plus trametinib in patients with previously treated $B R A F^{\mathrm{V} 600 \mathrm{E}_{-}}$ mutant metastatic non-small cell lung cancer: an open-label, multicentre phase 2 trial. Lancet Oncol 2016;17:984-93. 
84. Corcoran RB, Andre T, Atreya CE, et al. Combined BRAF, EGFR, and MEK inhibition in patients with $B R A F^{\mathrm{V} 600 \mathrm{E}}$-mutant colorectal cancer. Cancer Discov 2018;8:428-43.

85. Van Cutsem E, Huijberts S, Grothey A, et al. Binimetinib, encorafenib, and cetuximab triplet therapy for patients with $B R A F$ V600E-mutant metastatic colorectal cancer: safety lead-in results from the phase III BEACON colorectal cancer study. J Clin Oncol 2019;37:1460-9.

86. Sartore-Bianchi A, Trusolino L, Martino C, et al. Dualtargeted therapy with trastuzumab and lapatinib in treatment-refractory, KRAS codon 12/13 wild-type, HER2positive metastatic colorectal cancer (HERACLES): a proof-ofconcept, multicentre, open-label, phase 2 trial. Lancet Oncol 2016;17:738-46.

87. Raghav KPS, Overman MJ, Yu R, et al. HER2 amplification as a negative predictive biomarker for anti-epidermal growth factor receptor antibody therapy in metastatic colorectal cancer [abstract 3517]. JClin Oncol 2016;34:. [Available online at: https://ascopubs.org/doi/abs/10.1200/JCO.2016.34.15_ suppl.3517; cited 30 August 2019]

88. Meric-Bernstam F, Hurwitz H, Raghav KPS, et al. Pertuzumab plus trastuzumab for HER2-amplified metastatic colorectal cancer (MyPathway): an updated report from a multicentre, open-label, phase 2a, multiple basket study. Lancet Oncol 2019;20:518-30.

89. Nakagawara A. Trk receptor tyrosine kinases: a bridge between cancer and neural development. Cancer Lett 2001;169:107-14.

90. Martin-Zanca D, Hughes SH, Barbacid M. A human oncogene formed by the fusion of truncated tropomyosin and protein tyrosine kinase sequences. Nature 1986;319:743-8.

91. Creancier L, Vandenberghe I, Gomes B, et al. Chromosomal rearrangements involving the NTRK1 gene in colorectal carcinoma. Cancer Lett 2015;365:107-11.

92. Okamura R, Boichard A, Kato S, Sicklick JK, Bazhenova L, Kurzrock R. Analysis of NTRKalterations in pan-cancer adult and pediatric malignancies: implications for NTRK-targeted therapeutics. JCO Precis Oncol 2018;2018:.

93. Gatalica Z, Xiu J, Swensen J, Vranic S. Molecular characterization of cancers with NTRK gene fusions. Mod Pathol 2019;32:147-53.

94. Drilon A, Laetsch TW, Kummar S, et al. Efficacy of larotrectinib in TRK fusion-positive cancers in adults and children. N Engl J Med 2018;78:731-9. 